\title{
UNIVERSITYOF
}

FORWARD

THINKING

WESTMINSTER ${ }^{\text {网 }}$

WestminsterResearch

http://www.westminster.ac.uk/westminsterresearch

\section{An Extended NRBF Model for the Detection of Meat Spoilage}

Kodogiannis, V. and Alshejari, A.

This is a copy of the author's accepted version of a paper subsequently published in the proceedings of the 10th IEEE International Conference on Intelligent Systems IS'20, Varna, Bulgaria, 26 - 28 Aug 2020.

The final published version will be available online at:

https://doi.org/10.1109/IS48319.2020.9200181

(C) 2020 IEEE . Personal use of this material is permitted. Permission from IEEE must be obtained for all other uses, in any current or future media, including reprinting/republishing this material for advertising or promotional purposes, creating new collective works, for resale or redistribution to servers or lists, or reuse of any copyrighted component of this work in other works.

The WestminsterResearch online digital archive at the University of Westminster aims to make the research output of the University available to a wider audience. Copyright and Moral Rights remain with the authors and/or copyright owners. 


\section{An Extended NRBF Model for the Detection of Meat Spoilage}

\author{
Vassilis S. Kodogiannis \\ School of Computer Science \& Engineering \\ University of Westminster \\ London, United Kingdom \\ v.kodogiannis@westminster.ac.uk
}

\author{
Abeer Alshejari \\ Department of Mathematical Science \\ Princess Nourah bint Abdulrahman University \\ Riyadh, Saudi Arabia \\ AAAlshejari@pnu.edu.sa
}

\begin{abstract}
A rapid non-invasive detection of meat spoilage microorganisms, using Fourier transform infrared spectroscopy (FTIR) and Extended Normalized Radial Basis Function neural networks has been proposed in this paper. The objective is to associate simultaneously spectral data with microbiological data (log counts), for Total Viable Counts, Pseudomonas spp., Lactic Acid Bacteria and Enterobacteriaceae by predicting their microbiological population directly from FTIR spectra. The dimensionality reduction of spectral data has been addressed by the implementation of a fuzzy principal component algorithm, while results confirmed the superiority of the adopted scheme compared to multilayer perceptron models used recently in food microbiology.
\end{abstract}

Keywords-neural networks; expectation-maximization; fuzzy principal components analysis, meat spoilage, prediction.

\section{INTRODUCTION}

In the past few decades, meat industry has enormously flourished, due to the continuing growth of worldwide demand for improved food quality [1]. Beef, as one of the most commercially consumed muscle foods, although is a good food source for proteins and other vital nutrients it is also considered as an ideal substrate for the growth of pathogenic microorganisms and consequently spoilage. Currently, meat safety is mainly relied on regulatory inspection and sampling protocols and the majority of proposed chemical and microbiological detection methods are considered as timeconsuming processes [2]. Rapid and non-invasive methods based on analytical instrumental techniques, such as Fourier transform infrared spectroscopy (FTIR) [3], Raman spectroscopy [4], hyperspectral imaging [5] and electronic nose technology [6] have been considered for their potential in meat quality assessment.

Over the last few years, FTIR has been considered as a very important tool in food analysis. The application of chemometric-based techniques to associate FTIR spectral data with meat spoilage is not new and it has been investigated in the past [7]. FTIR spectral data collected directly from the surface of meat were used as biochemical interpretable "signatures", in an attempt to early microbial spoilage of chicken breast and rump steaks [8]. Partial least squares (PLS) and simple multilayer neural networks (MLP) models have been investigated to correlate FTIR spectral data with beef spoilage and its associated total viable bacteria counts (TVC) [9]. An advanced machine learning methodology based on adaptive fuzzy logic systems (AFLS) [10] has been proposed, utilizing the same dataset used earlier in [9].

The main objective of this paper is to associate FTIR spectral data with beef spoilage during aerobic storage at various temperatures $\left(0,5,10,15,20^{\circ} \mathrm{C}\right)$ utilizing an advanced learning-based decision support system, entitled $i$ MeatNet framework, based on Extended Normalized Radial Basis Function networks (ENRBF). Information related to FTIR spectra, as well as the correlated microbiological analysis from beef fillets (i.e. Total Viable Counts, Pseudomonas spp., Lactic Acid Bacteria and Enterobacteriaceae) was partly used in [9 \& $10]$. Due to the nature of FTIR spectral data, it is necessary to consider the use of a dimensionality reduction algorithm to reduce the problem of dimensionality with the minimum information lost. As PCA is vulnerable to outliers, in this paper an improvement of PCA is proposed by the fuzzification of the matrix data, in order to reduce the influence of the outliers. In the current study, a neural network scheme based on the Extended Normalized Radial Basis Function network (ENRBF) has been developed to predict the microbial load on meat surface. The Bayesian Ying-Yang (BYY) Expectation Maximization (EM) algorithm has been used together with novel splitting operations to determine network's size and parameter set. Results from ENRBF are compared against models based on MLP networks.

\section{FTIR SAMPLING AND ANALYSIS}

The FTIR experimental case was performed at the Agricultural University of Athens, Greece, and information related to FTIR spectra, as well as the correlated microbiological analysis (i.e. Total Viable Counts, Pseudomonas spp., Lactic Acid Bacteria and Enterobacteriaceae) from beef fillets, was provided to the first author for research purposes. A description of the experimental methodology as well as the related microbiological analysis of the meat samples is described in [9]. FTIR spectral information was used as a way to obtain metabolic "signatures" of beef fillet samples during storage in aerobic conditions at five different storage temperatures $\left(0,5,10,15\right.$, and $\left.20^{\circ} \mathrm{C}\right)$. Typical FTIR spectral data in the range of $1800-1000 \mathrm{~cm}^{-1}$ collected 
from fresh, semi-fresh and spoiled beef fillet samples stored at 0,10 and $20{ }^{\circ} \mathrm{C}$ respectively are shown in Fig. 1

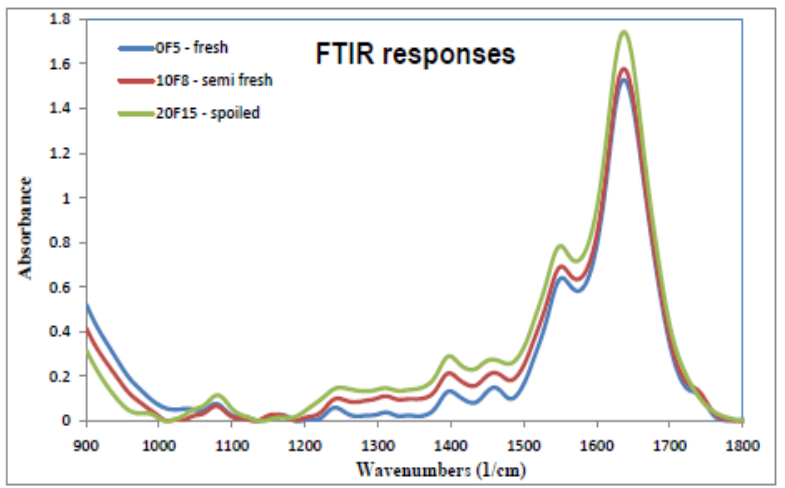

Figure 1. FTIR spectra collected from beef samples

Although the microbiological analysis for TVC prediction was performed on the entire group of 74 meat samples, such analysis for all specific microorganisms was accomplished only in 49 meat samples, as clearly shown from curves at Fig. 2. This inconsistency between the number of microbiological predictions of TVC and specific microorganisms represents a serious problem in applying learning-based systems in a homogeneous way.
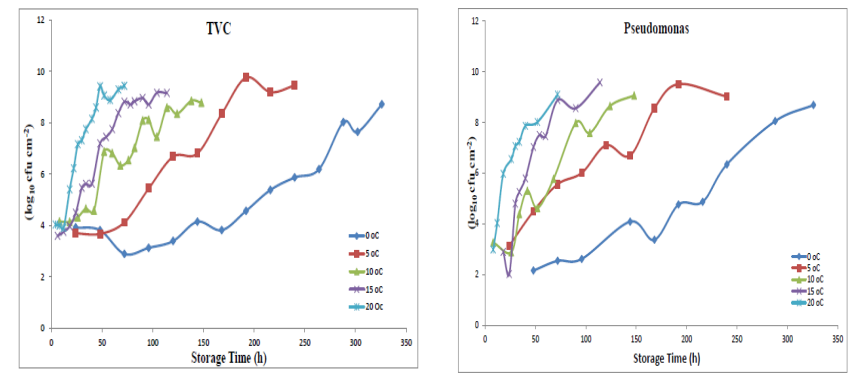

Figure 2. Growth curves of TVC \& Pseudomonas at various temperatures

The level of data quantity is generally considered as a main issue, because usually insufficient data will not lead to a robust prediction performance. In this work, an efficient data expansion technique has been utilized for the obtained small dataset to form a new "virtual" sample set, improving thus the accuracy of the proposed decision support system. Inspired by the way the Radial Basis Function (RBF) network approximates a nonlinear function through Gaussian local-basis functions, we have employed such a network to each "microorganism curve" defined from the experimental data. The aim is to associate each local-basis-function to each sample, and therefore easy then to generate "continuous virtual curves" that satisfy correlated experimental "microorganism curves". An RBF network using the regularized orthogonal least squares learning algorithm has been employed for this task [11]. Thus, for each microorganism case and for each temperature level, an individual RBF network has been constructed. As the real number of samples for each temperature level was limited, each RBF center was associated with a real sample. Then with a smaller sampling time, through a 2-inputs network, a "continuous growth curve" was obtained for each temperature level. The RBF inputs involved temperature level and sampling time-step, while the output was related to the specific microorganism predictions. Each "continuous growth curve" was verified against the real experimental samples. Based on these continuous "virtual" datasets, the missing microbiological information for the remaining 25 meat samples was retrieved.
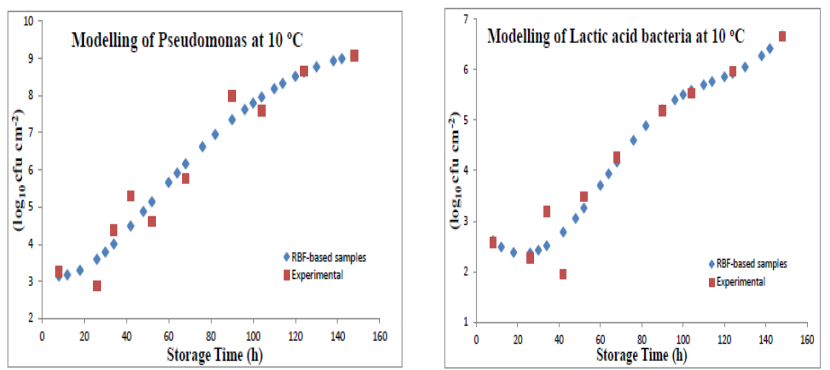

Figure 3. Modelling of microorganisms' growth curve via RBF networks

Fig. 3 illustrates a sample of these "virtual" datasets for the temperature of $10{ }^{\circ} \mathrm{C}$. The final dataset consisted of the "complete" microbiological predictions (i.e. 74 samples) as well as the sensory categorization was utilized for the development of the proposed prediction $i$ MeatNet framework. The initial FTIR dataset was divided into a training subset with approx. $80 \%$ of the data, and a testing subset with the remaining $20 \%$ (i.e. 14 samples). All testing samples were real experimental cases, whereas the "virtual" samples, which have been generated in order to fill the missing information from the microbiological analysis, were incorporated into the training datasets.

\section{DATA ANALYSIS}

In this work, FTIR spectral information was utilized to obtain metabolic "signatures" of beef fillet samples during storage in aerobic conditions at five different storage temperatures $\left(0,5,10,15\right.$, and $\left.20^{\circ} \mathrm{C}\right)$. Information from these spectra can be extracted in order to acquire metabolic fingerprints of beef fillets during storage at various temperatures. A principal component analysis (PCA) was then performed on this mean-centered spectral data. In this particular experimental case study, although the total variance $(100 \%)$ of the dataset was explained by 37 principal components (PCs), only the first five PCs were associated with the $97.85 \%$ of the total variance, as shown in Table I.

TABLE I. RESULTS FOR PCA AND FPCA SCHEMES

\begin{tabular}{|c|c|c|c|c|c|c|}
\hline PCs & \multicolumn{3}{c|}{ PCA } & \multicolumn{3}{c|}{ FPCA } \\
\cline { 2 - 7 } & E. value & $\begin{array}{c}\text { Prop. } \\
\text { \% }\end{array}$ & $\begin{array}{c}\text { Cum. } \\
\text { prop. 90 }\end{array}$ & E. value & Prop. & Cum. \\
\% & prop. 96 \\
\hline 1 & 6.060 & 47.874 & 47.874 & 2.670 & 57.405 & 57.405 \\
\hline 2 & 2.528 & 19.971 & 67.845 & 1.845 & 39.667 & 97.072 \\
\hline 3 & 1.824 & 14.409 & 82.254 & 0.063 & 1.354 & 98.426 \\
\hline 4 & 1.391 & 10.989 & 93.243 & 0.041 & 0.881 & 99.307 \\
\hline 5 & 0.583 & 4.606 & 97.849 & 0.019 & 0.408 & 99.715 \\
\hline
\end{tabular}


An alternative solution to improve PCA appears to be the fuzzification of the matrix data [12]. One approach toward the fuzzification of the matrix data is to consider the points that are isolated with respect to the first principal component. Fuzzy membership degrees can be introduced according to the distance to the first principal component. The first fuzzy principal components and the related fuzzy membership degrees could be determined through the usage of a fuzzy clustering algorithm. Elements with a high degree of membership in the ith cluster (i.e. close to the cluster's center) contribute significantly to the weighted average, while elements with a low degree of membership contribute negligibly. The algorithm could be considered as an extension of the fuzzy regression algorithm [13]. PCA and FPCA schemes were implemented in MATLAB, with the additional usage of PLS_Toolbox. Concerning the FPCA, of the same data set we have to remark that the results obtained are quite different. We can see that, for example, the first principal component explains $57.4 \%$ of the total variance and the second one 39.66; a two component model thus accounts for $97.07 \%$ of the total variance (as compared to $67.84 \%$ for PCA) and a three components model accounts for $98.42 \%$ (as compared to $82.25 \%$ for PCA) (Table I). Clearly, the first FPCA-derived components account for significantly more of the variance than the PCA counterparts. Thus, the first three principal components from the FPCA were extracted and utilized as inputs to the various simulation models applied on this particular dataset.

\section{EXTENDED NORMALISED RADIAL BASIS FUNCTION NETWORKS}

Locally active networks, like Radial Basis Function (RBF) networks, are less prone to dimensionality problems as each single neuron-node defines a multi-dimensional hyper-sphere in the input domain. This hyper-sphere is often defined through the use of a Gaussian activation function placed on the neuron. More frequently, instead of a single value utilized as the deviation parameter, a diagonal or full variance matrix is used. This alternative way provides differing levels of deviation along each axis of the input domain. Networks utilizing these types of neurons are often called Normalized Radial Basis Function (NRBF) networks. The identification scheme utilized in this paper is the Extended Normalized Radial Basis Function Network (ENRBF), which is an improvement of NRBF network [14].

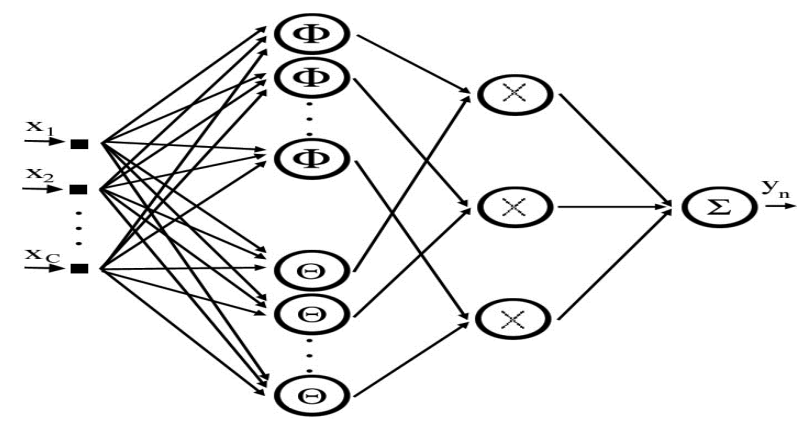

The ENRBF network replaces the linear combiner of the RBF with a series of local linear models as shown in Fig. 4. We propose a supervised training method for this scheme that is fully supervised as it incorporates the Bayesian Ying-Yang (BYY) method for parameter updating and uses a heuristic to determine the starting parameters of the network [15]. The BYY Expectation Maximization (EM) method treats the problem of optimization as one of maximizing the entropy between the original non-parametric data distribution based on Kernel estimates or user specified values and the parametric distributions represented by the network. This is achieved through the derivation of a series of EM update equations using a series of entropy functions as the Q function or log-likelihood function. The ENRBF network can be represented by the following set of equations.

$$
E(z \mid x, \Theta)=\frac{\sum_{j=1}^{K}\left(W_{j}^{T} x+c_{j}\right) p\left(x \mid j, \theta_{j}\right)}{\sum_{j=1}^{K} p\left(x \mid j, \theta_{j}\right)}
$$

where $z$ is the output of the network $z \in Z, x$ is an input vector $x \in X, \Theta=[W, c, \theta]$ are the network parameters and $\theta=[m, \Sigma]$ are the parameters of the Gaussian activation functions given by

$$
p\left(x \mid j, \theta_{j}\right)=\exp \left\{-\frac{1}{2}\left(x-m_{j}\right)^{T} \Sigma_{j}^{-1}\left(x-m_{j}\right)\right\}
$$

The BYY method attempts to maximize the degree of agreement between the expected value of $z$ from the network and the true value of $z$ from the training data. It is guaranteed to lead to a local optimum and unlike the original EM algorithm for learning the parameters of Gaussian functions this method encourages coordination between the input and output domains. Like the EM algorithm, this method is very fast in terms of the number of iterations needed for the parameters to converge. It is this speed of convergence that makes the proposed technique feasible. However, as BYY is an EM based technique it is still susceptible to locally maximal values. The Split and Merge EM (SMEM) concept for Gaussian Mixture Models (GMM) proposed initially by Ueda, has been applied to the ENRBF scheme [15]. The original SMEM algorithm is able to move neurons from over populated areas of the problem domain to underrepresented areas by merging the over populated neurons and splitting the under-populated. The use of Eigenvectors to split along the axis of maximum divergence instead of randomly as in original SMEM is an alternative methodology. The SMEM algorithm suffers from the fact that before terminating all possible combinations of Split and Merge operations must be examined. Although many options can be discounted, the training still increases exponentially with network size and again suffers from the problems inherent with k-means and basic EM. A splitting technique that overcomes these problems has been proposed by one of the authors [15].

Figure 4. ENRBF scheme 


\section{RESULTS \& DISCUSSION}

The final FTIR dataset, consisted of 74 beef patterns, include information from the various storage temperatures, the sampling times and the first three PCs from the FPCA scheme. The ENRBF scheme has been implemented through MATLAB and its structure consists of an input layer which in this current research study contains five input nodes (i.e. storage temperature, sampling time, and the values of the first three PCs). The output node corresponds to the related microbiological attribute. As the related microbiological analysis involved four attributes (i.e. total viable counts (TVC), Pseudomonas app. Lactic acid bacteria (LAB) and Enterobacteriaceae), a decision support system, " $i$ MeatNet", has been designed in such way in order to accommodate all relevant information. Its overall schematic diagram which is illustrated at Fig. 5 shows a parallel system consisted of four ENRBF module units, which provide the individual microbiological predictions.

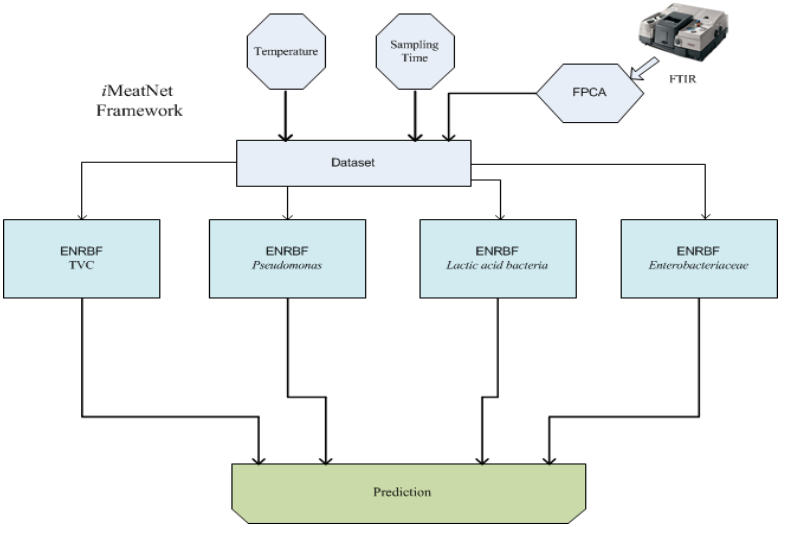

Figure 5. " $i$ MeatNet" decision support framework

The real challenge in this paper is to propose a new learning-based structure which could be considered as a benchmark method towards the development of efficient intelligent methods in food quality analysis. For this reason, ENRBF's prediction results are compared with those obtained by an MLP identification model. Such scheme has become a popular modelling technique in food science and technology in recent years.

\section{A. Prediction of microbiological features}

Results revealed that the identification accuracy of the ENRBF model was very satisfactory in the prediction of TVCs, indicating the advantage of this approach in tackling complex, nonlinear problems, such as meat spoilage. The plot of predicted (via ENRBF) vs. observed TVCs is illustrated in Fig. 6 , and shows a very good distribution around the line of equity $(\mathrm{y}=\mathrm{x})$, with all the data included within the $\pm 1 \log$ unit area. A close inspection reveals more information regarding the "behavior" of specific beef samples. Two samples, as shown from Fig. 6a, are in the border line of the $\pm 1 \log$ unit area and they are associated to the semi-fresh "10F7" and the spoiled "5F9" samples. "10F7" corresponds to a beef fillet, stored at $10^{\circ} \mathrm{C}$ and collected after $52 \mathrm{~h}$ of storage, while " $5 \mathrm{F9}$ " was stored at $5 \mathrm{oC}$ and collected after $192 \mathrm{~h}$ of storage. Two fresh samples (i.e. "0F5" and "5F3") are close to the border line at
Fig. 6a. "0F5" corresponds to a beef fillet, stored at $0 \mathrm{oC}$ and collected after $96 \mathrm{~h}$ of storage, while " $5 \mathrm{~F} 3$ " was stored at $5^{\circ} \mathrm{C}$ and collected after $48 \mathrm{~h}$ of storage. It seems that all these "suspicious" cases occur at low temperatures. TVC growth curves at Fig. 2, reveal that both $0^{\circ} \mathrm{C}$ and $5^{\circ} \mathrm{C}$ curves have an initial flat response, which could justify such "suspicious" behavior. A possible way to overcome this problem could be to broaden the training dataset, especially for low temperatures.

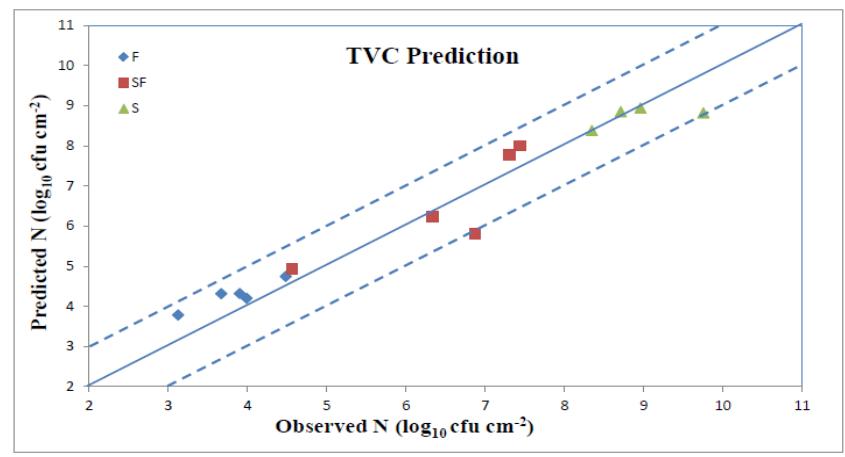

Figure 6. Performance of ENRBF prediction model for TVC

In addition, an MLP was constructed with two hidden layers (with 12 and 6 nodes respectively) for the prediction of TVCs. The performance of the ENRBF and MLP models in predicting TVCs in beef samples in terms of statistical indices is presented in Table II.

TABLE II. PERFORMANCE OF IDENTIFICATION MODELS FOR TVC PREDICTION

\begin{tabular}{|l|c|c|c|c|c|}
\hline TVC & Fresh & $\begin{array}{c}\text { Semi- } \\
\text { fresh }\end{array}$ & Spoiled & $\begin{array}{c}\text { Overall } \\
\text { (ENRBF) }\end{array}$ & $\begin{array}{c}\text { Overall } \\
\text { (MLP) }\end{array}$ \\
\hline $\begin{array}{c}\text { Stat. } \\
\text { Indices }\end{array}$ & ENRBF & ENRBF & ENRBF & & \\
\hline$M S E$ & 0.2287 & 0.3592 & 0.2214 & 0.2733 & 0.5757 \\
\hline$R M S E$ & 0.4783 & 0.5994 & 0.4706 & 0.5227 & 0.7587 \\
\hline$M A P E$ & 0.1213 & 0.0777 & 0.0293 & 0.0794 & 0.1075 \\
\hline$B_{f}$ & 1.1195 & 1.0058 & 0.9798 & 1.0372 & 1.0343 \\
\hline$A_{f}$ & 1.1195 & 1.0820 & 1.0309 & 1.0802 & 1.1108 \\
\hline$S E P \%$ & 12.4714 & 9.2144 & 5.2620 & 8.3669 & 12.144 \\
\hline
\end{tabular}

Bias factor $\left(B_{f}\right)$ is a multiplicative factor that compares model predictions and is used to determine whether the model overor under-predicts the response time of bacterial growth. Based on the calculated values of $B_{f}$, it can be concluded that the ENRBF model over-estimated total viable counts in fresh samples $\left(B_{f}>1\right)$, whereas for spoiled samples, underestimation of microbial population was evident $\left(B_{f}<1\right)$ and almost perfect for semi-fresh samples (1.0058). The accuracy factor $A_{f}$ is a simple multiplicative factor that indicates the spread of results about the prediction. In the case of ENRBF, the values of $A_{f}$ indicated that the predicted total viable counts were $11.95 \%, 8.2 \%$, and $3.09 \%$ different (either above or below) from the observed values for fresh, semi-fresh, and spoiled meat samples, respectively. The relevant overall figures indicate again a better performance for ENRBF compared to MLP model. Overall, with MAPE 7.94\% for ENRBF against 
$10.75 \%$ for MLP, the proposed "localized-learning" model managed to achieve a better performance. The value of standard error of prediction (SEP) was $8.367 \%$ for the overall samples, indicating good performance of the network for microbial count predictions.

An additional ENRBF model has been utilized for the case of Pseudomonas spp. The related plot of predicted (via ENRBF) vs. observed microorganism is illustrated in Fig. 7, and shows a good distribution around the line of equity $(\mathrm{y}=\mathrm{x})$, with all the data (expect one) included within the $\pm 1 \log$ unit area.

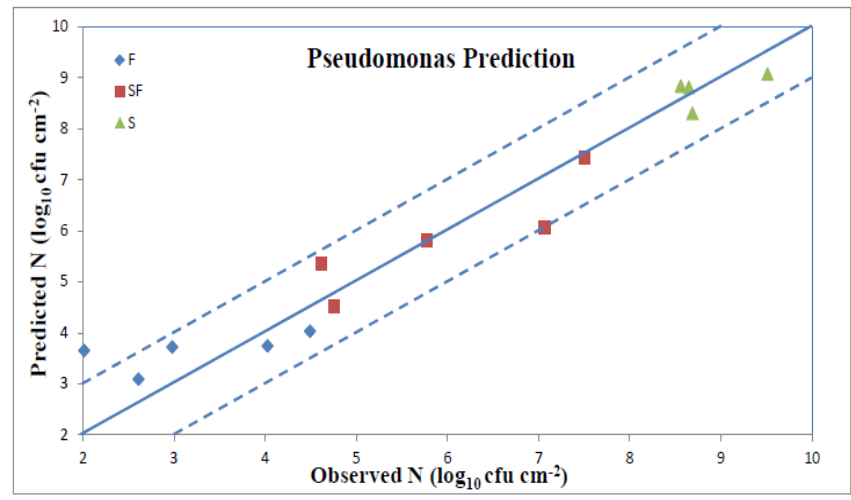

Figure 7. Performance of ENRBF prediction model for Pseudomonas

One fresh sample ("15F5"), as shown from Fig. 7, is clearly outside of the $\pm 1 \log$ unit area. " $15 \mathrm{~F} 5$ " corresponds to a beef fillet, stored at $15^{\circ} \mathrm{C}$ and collected after $24 \mathrm{~h}$ of storage. Similarly to ENRBF case, an MLP model was implemented for the prediction of Pseudomonas, and both performances in terms of statistical indices, are presented in Table III. The relative high SEP index for both ENRBF and MLP indicate high-nonlinearity behavior for Pseudomonas, especially for the case of fresh samples.

TABLE III. PERFORMANCE OF IDENTIFICATION MODELS FOR PSEUDOMONAS PREDICTION

\begin{tabular}{|l|c|c|c|c|c|}
\hline Pseudomonas & Fresh & $\begin{array}{c}\text { Semi- } \\
\text { fresh }\end{array}$ & Spoiled & $\begin{array}{c}\text { Overall } \\
\text { (ENRBF) }\end{array}$ & $\begin{array}{c}\text { Overall } \\
\text { (MLP) }\end{array}$ \\
\hline Stat. Indices & ENRBF & ENRBF & ENRBF & & \\
\hline$M S E$ & 0.7512 & 0.3257 & 0.1104 & 0.4162 & 0.7691 \\
\hline$R M S E$ & 0.8667 & 0.5707 & 0.3323 & 0.6451 & 0.8770 \\
\hline$M A P E$ & 0.2836 & 0.0742 & 0.0354 & 0.1379 & 0.1422 \\
\hline$B_{f}$ & 1.1749 & 0.9885 & 0.9899 & 1.0518 & 0.9924 \\
\hline$A_{f}$ & 1.2627 & 1.0771 & 1.0364 & 1.1276 & 1.1423 \\
\hline$S E P \%$ & 26.8755 & 9.6014 & 3.7544 & 11.1158 & 15.1113 \\
\hline
\end{tabular}

Lactic acid bacteria (LAB) utilize glucose at the meat surface. When the glucose is exhausted, they begin to metabolize amino acids in the meat, and start to produce the offensive odors associated with spoilage. The activity of organic acids produced by LAB is primarily associated with the chemical state of the acid, which is affected by the level of $\mathrm{pH}$. In this particular case, $\mathrm{pH}$ had been set to 5.7. The plot of predicted (via ENRBF) vs. observed LAB is illustrated in Fig. 8 , and shows a good distribution around the line of equity $(y=x)$, with all the data (expect one) included within the $\pm 1 \log$ unit area.

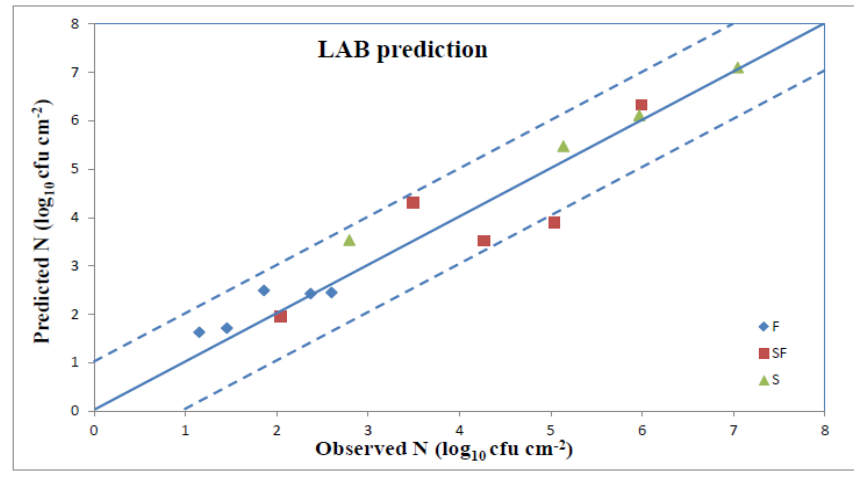

Figure 8. Performance of ENRBF prediction model for Lactic Acid Bacteria

The plot of predicted (via ENRBF) vs. observed LAB is illustrated in Fig. 8, and shows a good distribution around the line of equity $(\mathrm{y}=\mathrm{x})$, with all the data (expect one) included within the $\pm 1 \log$ unit area. Model's performance is confirmed by checking the statistical analysis, shown at Table IV. Both fresh and semi-fresh samples have high SEP, MAPE and $A_{f}$ indices compared to those associated to spoiled samples. However the overall SEP was kept at $14.76 \%$. A similar, but inferior in terms of accuracy, performance was obtained with the use of a two-hidden MLP network.

TABLE IV. PERFORMANCE OF IDENTIFICATION MODELS FOR LACTIC ACID BACTERIA PREDICTION

\begin{tabular}{|l|c|c|c|c|c|}
\hline \multicolumn{1}{|c|}{ LAB } & Fresh & Semi-fresh & Spoiled & $\begin{array}{c}\text { Overall } \\
\text { (ENRBF) }\end{array}$ & $\begin{array}{c}\text { Overall } \\
\text { (MLP) }\end{array}$ \\
\hline Stat. Indices & ENRBF & ENRBF & ENRBF & & \\
\hline$M S E$ & 0.1457 & 0.5329 & 0.1726 & 0.2916 & 0.4767 \\
\hline$R M S E$ & 0.3817 & 0.7300 & 0.4154 & 0.5400 & 0.6904 \\
\hline$M A P E$ & 0.2040 & 0.1478 & 0.0910 & 0.1517 & 0.1975 \\
\hline$B_{f}$ & 1.1671 & 0.9552 & 1.0864 & 1.0645 & 1.0615 \\
\hline$A_{f}$ & 1.1950 & 1.1647 & 1.0864 & 1.1523 & 1.2001 \\
\hline$S E P \%$ & 20.2213 & 17.5205 & 7.9331 & 14.7621 & 18.8736 \\
\hline
\end{tabular}

Statistics at Table IV confirmed such similarity with ENRBF, although overall results reveal the deficiencies of globallearning schemes such as MLPs.

Enterobacteriaceae bacterium was the last experiment in this research study. The plot of predicted (via ENRBF) vs. this specific observed microorganism is illustrated in Fig. 9, and shows an excellent distribution around the line of equity $(y=x)$, with all the data (expect one) included within the $\pm 1 \log$ unit area. The semi-fresh sample "15F10" which is placed outside the $\pm 1 \log$ unit area, is associated with a beef fillet, stored at $15^{\circ} \mathrm{C}$ and collected after $54 \mathrm{~h}$ of storage. The performance of the ENRBF model in predicting Enterobacteriaceae in beef samples in terms of statistical indices is presented in Table V. These results are in agreement with Fig. 9, and reveal overall small RMSE and SEP, while the value of $B_{f}$ is almost optimal. Statistics at Table $\mathrm{V}$ summarize also the prediction performance for MLP model developed for this particular case study. 
In summary, ENRBF and MLP networks utilize a different "build philosophy" for their architectures. An MLP network, due to the use of sigmoid activation functions in its structure, utilizes a "global-learning strategy" compared to ENRBF's "local-learning strategy" models that utilize Gaussian activation functions.

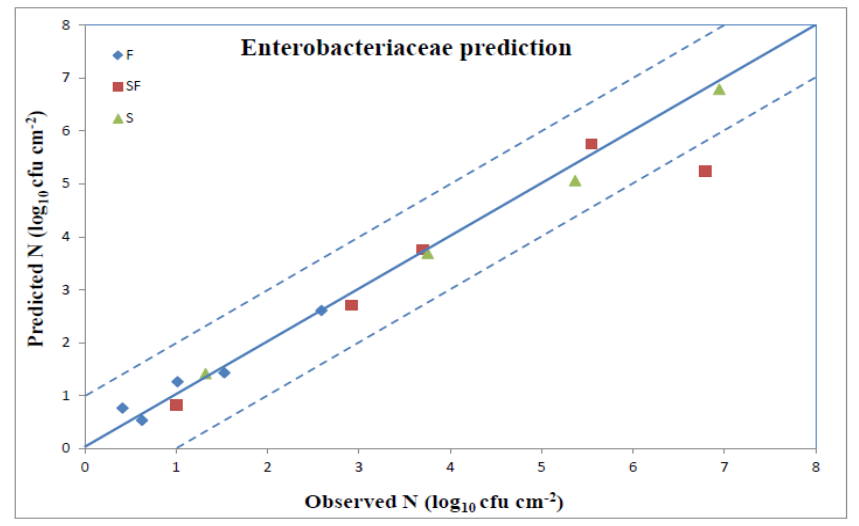

Figure 9. Performance of ENRBF prediction model for Enterobacteriaceae

In MLP model, all normalized inputs are fed to the hidden layer, while in the case of ENRBF each input is decomposed through Gaussians functions.

TABLE V. PERFORMANCE OF IDENTIFICATION MODELS FOR ENTEROBACTERIACEAE PREDICTION

\begin{tabular}{|l|c|c|c|c|c|}
\hline $\begin{array}{c}\text { Entero- } \\
\text { bacteriaceae }\end{array}$ & Fresh & Semi-fresh & Spoiled & $\begin{array}{c}\text { Overall } \\
\text { (ENRBF) }\end{array}$ & $\begin{array}{c}\text { Overall } \\
\text { (MLP) }\end{array}$ \\
\hline Stat. Indices & ENRBF & ENRBF & ENRBF & & \\
\hline$M S E$ & 0.0411 & 0.5027 & 0.0323 & 0.2034 & 0.4809 \\
\hline$R M S E$ & 0.2027 & 0.7090 & 0.1796 & 0.4510 & 0.6934 \\
\hline$M A P E$ & 0.2637 & 0.1070 & 0.0423 & 0.1445 & 0.2591 \\
\hline$B_{f}$ & 1.1349 & 0.9082 & 0.9934 & 1.0089 & 1.0411 \\
\hline$A_{f}$ & 1.2377 & 1.1246 & 1.0430 & 1.1390 & 1.2614 \\
\hline$S E P \%$ & 16.4273 & 17.7592 & 4.1329 & 14.5097 & 22.3090 \\
\hline
\end{tabular}

This localization spread through these functions, is one advantage against the classic MLP structure. In addition, the usage of BYY-EM learning scheme contributed to a much faster training compared to the classic gradient descent method.

\section{CONCLUSIONS}

In conclusion, this simulation study demonstrated the effectiveness of the detection approach based on FTIR spectroscopy which in combination with an appropriate machine learning strategy could become an effective tool for monitoring meat spoilage during aerobic storage at various temperatures. The system consists of four advanced neural network based identification units, which provide the individual microbiological predictions. In the current study, a fuzzy PCA scheme has been implemented through the fuzzification of the matrix data, taking into consideration the first principal component. Results and comparison with MLP networks revealed an overall very satisfactory accuracy. There is need to explore further the use of advanced intelligent systems, and this paper has attempted for the first time to associate FTIR spectra with such systems. The ENRBF performance although very convincing, discloses however a second open problem, that is the need to have or "create" large training datasets, even with the presence of small amount of real experimental data.

\section{REFERENCES}

[1] M. Gagaoua, B. Picard, "Current Advances in Meat Nutritional, Sensory and Physical Quality Improvement”, Foods, vol. 9, No. 3, pp. 321, 2020

[2] I.H., Cho, K. Seockmo, "Current Technical Approaches for the Early Detection of Foodborne Pathogens: Challenges and Opportunities", Intern. journal of molecular sciences, vol. 18, No.10, pp. 2078, 2017.

[3] D.J.M. Mouwen, A. Hörman, H. Korkeala, A. Alvarez-Ordóñez, M. Prieto, "Applying Fourier-transform infrared spectroscopy and chemometrics to the characterization and identification of lactic acid bacteria”, Vibrational Spectroscopy, vol. 56, No. 2, pp. 193-201, 2011.

[4] Q. Wang, S.M. Lonergan, C. Yu, "Rapid determination of pork sensory quality using Raman spectroscopy”, Meat Science, vol. 91, No. 3, pp. 232-239, 2012

[5] F. Tao, Y. Peng, "A method for non-destructive prediction of pork meat quality and safety attributes by hyperspectral imaging technique", J Food Eng., vol. 126, pp. 98-106, 2014.

[6] V.S. Kodogiannis, "Application of an Electronic Nose Coupled with Fuzzy-Wavelet Network for the Detection of Meat Spoilage", Food Bioprocess Technol., vol. 10, pp. 730-749, 2017.

[7] M.S.Ammor, A. Argyri, G.-J.E. Nychas, "Rapid monitoring of the spoilage of minced beef stored under conventionally and active packaging conditions using Fourier transform infrared spectroscopy in tandem with chemometrics", Meat Science, vol. 81, No. 3, pp. 507-514, 2009

[8] D.I. Ellis, D. Broadhurst, D.B. Kell, J.J. Rowland, R. Goodacre, "Rapid and quantitative detection of the microbial spoilage of meat by Fourier transform infrared spectroscopy and machine learning", Appl. Environ. Microbiol, vol. 68, pp. 2822-2828, 2002

[9] A.A. Argyri, E.Z. Panagou, P.A. Tarantilis, M. Polysiou, \& G.-J. E. Nychas, "Rapid qualitative and quantitative detection of beef fillets spoilage based on Fourier transform infrared spectroscopy data and artificial neural networks", Sensors and Actuators B, vol. 145, pp. 146154,2010

[10] V. Kodogiannis, T. Pachidis, E. Kontogianni, “An intelligent based decision support system for the detection of meat spoilage", Engineering Applications of Artificial Intelligence, vol. 34, pp. 23-36, 2014.

[11] M. Amina, E.Z. Panagou, V.S. Kodogiannis, G.-J.E. Nychas, "Wavelet Neural Networks for modelling high pressure inactivation kinetics of Listeria monocytogenes in UHT whole milk", Chemometrics and intelligent laboratory systems, vol. 103, No. 2, pp. 170-183, 2010.

[12] K. Szczepaniak, C. Sârbu, A. Astel, E. Raińska, M. Biziuk, O. Culicov, M.V. Frontasyeva, P. Bode, "Assessment of the impact of a phosphatic fertilizer plant on the adjacent environment using fuzzy logic", Central European Journal of Chemistry, vol. 4, No. 1, pp. 29-55, 2006.

[13] V.S. Kodogiannis, I. Petrounias and E. Kontogianni, "Identification of meat spoilage by FTIR spectroscopy and neural networks," 2014 International Joint Conference on Neural Networks (IJCNN), Beijing, 2014, pp. 1644-1651.

[14] E. Wadge, The use of EM-Based neural network schemes for modelling and classification, PhD Thesis, Westminster University, 2005

[15] V.S. Kodogiannis, E. Wadge, "The Use of Gas-Sensor Arrays to Diagnose Urinary Tract Infections", Int. Journal of Neural Systems, vol. 15 , No. 5, pp. 363-376, 2005. 